\title{
SARS-CoV-2 vaccines and autoimmune diseases amidst the COVID-19 crisis
}

\author{
Tsvetelina Velikova' ${ }^{1}$ - Tsvetoslav Georgiev ${ }^{2,3}$ (1)
}

Received: 22 December 2020 / Accepted: 13 January 2021 / Published online: 30 January 2021

(c) The Author(s), under exclusive licence to Springer-Verlag GmbH, DE part of Springer Nature 2021

\begin{abstract}
Coronavirus disease 2019 (COVID-19) pandemic has become challenging even for the most durable healthcare systems. It seems that vaccination, one of the most effective public-health interventions, presents a ray of hope to end the pandemic by achieving herd immunity. In this review, we aimed to cover aspects of the current knowledge of severe acute respiratory syndrome coronavirus 2 (SARS-CoV-2) vaccines and vaccine candidates in the light of autoimmune inflammatory diseases (AIIDs) and to analyze their potential in terms of safety and effectiveness in patients with AIIDs. Therefore, a focused narrative review was carried out to predict the possible implications of different types of SARS-CoV-2 vaccines which confer distinct immune mechanisms to establish immune response and protection against COVID-19: whole virus (inactivated or weakened), viral vector (replicating and non-replicating), nucleic acid (RNA, DNA), and protein-based (protein subunit, virus-like particle). Still, there is uncertainty among patients with AIIDs and clinicians about the effectiveness and safety of the new vaccines. There are a variety of approaches towards building a protective immunity against SARS-CoV-2. Only high-quality clinical trials would clarify the underlying immunological mechanisms of the newly implemented vaccines/ adjuvants in patients living with AIIDs.
\end{abstract}

Keywords COVID-19 · SARS-CoV-2 · COVID-19 vaccines · Autoimmune diseases · Messenger RNA · Vaccination · Rheumatic diseases

\section{Introduction}

Coronavirus disease 2019 (COVID-19) remains a global threat to humanity that calls for experts, policy-makers, and governments to confront several aspects that go well beyond the health and well-being consequences of this pandemic. While the pandemic's immediate health implications unfold, we demand studies and interventions to be reorganized to

Tsvetoslav Georgiev

tsetso@medfaculty.org

Tsvetelina Velikova

tsvelikova@medfac.mu-sofia.bg

1 Department of Clinical Immunology, Medical Faculty, University Hospital "Lozenetz", Sofia University St. Kliment Ohridski, 1 Kozyak Str., 1407 Sofia, Bulgaria

2 First Department of Internal Medicine, Faculty of Medicine, Medical University-Varna, 55 Marin Drinov Str., Varna 9002, Bulgaria

3 Clinic of Rheumatology, University Hospital "St. Marina", 1 Hristo Smirnenski Blvd., 9010 Varna, Bulgaria mitigate the risk and create continuity and improved resilience for potential recovery [1]. Moreover, COVID-19 has become challenging even for the most durable healthcare systems. To contain the severe acute respiratory syndrome coronavirus 2 (SARS-CoV-2) spread, drastic restrictive measures were taken around the globe. Lockdowns, at the cost of economic slowdowns and restriction of human freedoms, seem to be one of the few effective interventions [2].

Due to the pandemic, we observe tensions of the ultimate social determinant of health, causing a wide range of issues from health policy limitations to concerns in the distribution and access to health care. Reorienting healthcare services to COVID-19 management in the already over-extended and underfunded health systems has limited their ability to hold other disease burdens. Furthermore, halting routine procedures and interventions, follow-up, and immunization programs leads to outbreaks of preventable transmissible diseases, raised cancer incidence, and an increased number of complicated medical disorders in the late stages [1].

At the same time, based on mounting evidence (or not), treatment protocols were developed, most commonly 
including corticosteroids, anticoagulants, antiviral drugs, and immunotherapeutics $[3,4]$. However, the expected second wave, characteristic of respiratory infections, surprised humanity again and inflicted more significant damage from the first. The optimism was beginning to vanish. Importantly, achieving herd immunity to control a pandemic through natural infection will undoubtedly lead to the deaths of millions [5]. In this moment of despair and lack of control over the infection, at the dawn of the pandemic, the pure discovery nature of humanity gave us a ray of hope. It seems that we may combat the problem through one of the most effective public-health interventions-vaccination [6]. While COVID-19 vaccinations offer the promise of a return to the usual standards of healthcare, the vaccine-based solution needs sufficient population coverage. It requires acceptable policy, operational, and logistical actions. However, at the current phase of the COVID-19 pandemic, it is essential to discriminate between those who will benefit most from the vaccination and those who may have reasonable concerns regarding vaccination. Besides, it is critical to distinguish the entire dissimilar group of people experiencing vaccine hesitancy and vaccine denial [7].

Most of the candidates in the portfolio of the COVID-19 Vaccine Global Access Facility (COVAX) are based on nextgeneration technologies that could be developed and skilled up more quickly than used-to-be conventional vaccines [8]. In line with this, patients with chronic autoimmune inflammatory diseases (AIIDs), specifically rheumatic diseases and rheumatologists, have their concerns about vaccination.

We aimed to review the current knowledge of SARS$\mathrm{CoV}-2$ vaccines and vaccine candidates in the light of AIIDs and analyze their potential to be effective and safe in patients with AIIDs. A focused narrative review was carried out to predict the possible implications of different types of SARS-CoV-2 vaccines, which confer distinct immune mechanisms to establish immune response and protection against COVID-19.

\section{Search strategy}

A modified form of a narrative review was chosen that adhered to recent recommendations for writing a biomedical narrative review [9] where a search through scientific databases was combined with solid evidence from studies on vaccine effectiveness and safety in patients with autoimmune diseases. Initially, a thorough literature search was carried out in the bibliographic databases Medline (PubMed) and Scopus. Both MeSH and relevant free-text terms were used, as follows: ("COVID-19" OR "SARS-CoV-2") AND ("autoimmune" OR "rheumatic") AND ("vaccine*" OR "mRNA"). Our search was confined to articles published from 1 January 2020 to 7 January 2021. Secondly, due to the solid experience with vaccinations against other infectious diseases in the rheumatic population and the scarce information about COVID-19 vaccines, older publications were also included. Relevant data were also derived from preprints identified using the search engine Google Scholar. Information from advisory committee meetings was identified based on the prior knowledge of the authors. Finally, references of retrieved publications were further hand-searched for supplements.

\section{COVID-19 and patients with autoimmune inflammatory diseases}

Autoimmune inflammatory diseases represent a heterogeneous group of over eighty inflammatory disorders with an aberrant immune response to self [10]. The broad spectrum of interactions between AIIDs and SARS-CoV-2 infection are unpredictable, and the mechanism underlying this relationship remains poorly understood. Like in other viral infections, COVID-19 could trigger an AIID flare. Nevertheless, beyond rheumatologists' expectations, COVID-19 is linked to autoimmune and autoinflammatory sequelae raising questions about its long-term immune-mediated consequences and complications [11]. Importantly, musculoskeletal complaints, cytopenias, the pattern of lung involvement, vasculitic events, secondary thrombophilia, and glucocorticoid responsiveness approximate the manifestations of AIIDs [12].

Based on the latest data from the EULAR COVID-19 database, as of 1 December 2020, approximately 3590 patients with rheumatic diseases were reported to have contracted SARS-CoV-2 [13], representing only the tip of the iceberg. Unique to the population with AIID is the underlying autoimmunity, common comorbidities (cardiovascular and gastrointestinal disorders, kidney and lung diseases, infections, neoplasms, and depression) [14], and ongoing therapy with immunosuppressive and/or glucocorticoids $[4,12]$. The heterogeneity of AIIDs and their disease activity defines the great diversity of therapeutic agents ranging from immunomodulators such as antimalarials through biologics and new target synthetic agents and finally to potent immunosuppressive drugs such as alkylating agents, fully compromising the immune system. Notably, the administered therapeutic agents bring another variable to the anyway complicated equation weighing the potential benefits and detriments of vaccination.

In recent decades, biological and new target synthetic agents have tremendously improved and modified the course of AIID and significantly improved the disease outcomes. On the other hand, they have led to some changes in an "unfavorable" direction, generally increasing the risk of infections. Nevertheless, numerous high-quality studies have 
already found that the risk of contracting SARS-CoV-2 and poor COVID-19 outcomes of patients with the most common AIID is similar to the general population $[15,16]$. In the case of immunosuppression, the response to some vaccines and the efficacy resulting in immunogenicity, however, has become unpredictable, especially in the case of B cell depleting therapies [17]. Those concerns were recently addressed in the European League Against Rheumatism (EULAR) recommendations. Ahead of time and events, EULAR has stated that immunization with non-live vaccines could be carried out during the use of glucocorticoids or anti-rheumatic drugs [18]. Is that the case with the new COVID-19 vaccines?

Although preliminary data showed that AIIDs themselves did not pose an increased risk of SARS-CoV-2 infection and severe disease $[15,16]$, there is a complexity of disease-disease and drug-disease interactions characteristic of AIIDs that need special consideration. AIID-induced organ damage, coexisting chronic cardiovascular, metabolic, and respiratory comorbidities, predisposition to bacterial infections, B cell depletion therapies, and the use of high-dose glucocorticoids could increase the risk of poor outcome [19]. Therefore, COVID-19 prevention strategies should be prioritized in the specific population of patients with AIIDs, and vaccination against SARS-CoV-2 is thought to be one of the most promising approaches. Nevertheless, their safety and efficacy in the light of emerging evidence should be primarily and consistently analyzed.

\section{Vaccines recommendations and safety concerns in patients with autoimmune diseases}

Theoretically, autoimmunity may potentially be triggered by vaccines. Immune mechanisms such as antigen presentation, cytokines production, anti-idiotypic networks, bystander activation, epitope spreading, and polyclonal activation of B cells are involved in both anti-infectious immune response and autoreactivity [20,21]. It is essential to emphasize that to develop autoimmunity, in addition to the aforementioned mechanisms, autoreactive $\mathrm{T}$ and $\mathrm{B}$ cells have to be involved and self-epitopes to be exposed during the infection. This is the case in the minority of the infections. Nevertheless, antinuclear antibodies and autoimmune cell-mediated responses are common during and after viral infections. However, this transient autoreactive positivity is not followed by any symptoms or clinical consequences [22, 23]. Noteworthy, autoreactivity is not equal to autoimmunity due to various and sufficient control mechanisms that regulate the immune responses. Besides, there is strong evidence that autoimmune disease development depends on many other factors, except for vaccines. Since autoimmune diseases occur in the absence of vaccines, it is not feasible to assume that vaccines cause autoimmunity alone [24].

Furthermore, there is substantial evidence that infectious agents are significantly linked to causing autoimmunity [25]. The possible positive association between vaccines and autoimmunity was mostly based on anecdotal cases, case reports, and uncontrolled observational studies [24]. For example, Campylobacter was linked to Guillain-Barre syndrome (GBS) [26], influenza virus-multiple sclerosis [27], Coxsackie virus-diabetes type 1 [28], Human parvovirus B19-rheumatoid arthritis [29], and the list is not exhaustive.

Since natural infection can result in autoimmune disorders, it is rational to question whether vaccines can also induce autoimmunity. Or whether the vaccination can increase the prevalence of autoimmune disorders in some susceptible individuals. Multiple studies have investigated various vaccines. However, no autoimmune disorders have been demonstrated as caused by vaccines so far [24]. Talking for GBS, for example, it was estimated that the relative risk for the development of the syndrome is tenfold higher after natural flu infection compared to the influenza vaccine application, or in other words - one additional case per one million people vaccinated. Besides, this estimation excludes other known severe influenza-related complications [20,30].

Significantly, uncontrolled infection-induced autoreactive mechanisms may transform into autoimmunity in individuals with a strong genetic predisposition [31, 32]. In line with this, some individuals indeed develop autoimmune diseases following vaccines, but these are rare. Nevertheless, experts are exploring autoimmunity issues related to vaccines [24]. However, the majority of people can vaccinate without any risk of autoimmunity onset [33]. Three main issues need to be addressed about vaccines and patients living with autoimmune diseases:

- Are there any contraindicated vaccines?

- Will vaccines be sufficiently effective while on immunosuppressive treatment?

- Could vaccines cause an AIID flare?

Only a few vaccines should be avoided in patients with autoimmune diseases. For rheumatic patients on glucocorticosteroids or disease-modifying antirheumatic drugs (such as cyclophosphamide, methotrexate, azathioprine, tumor necrosis factor inhibitors, etc.), the use of live attenuated viruses is not recommended (e.g., flu shot, rotavirus vaccine for babies of female patients on immunosuppressants), although some vaccines, such as measles, mumps, and rubella (MMR), and herpes zoster vaccines, can be used with cautious when the benefits outweigh the risks in patients on low doses of immunosuppressants [18]. 
However, patients with autoimmune inflammatory rheumatic diseases can safely obtain all the benefits of the inactivated vaccines, or those based on recombinant proteins or toxoids (i.e., "killed" flu shot, pneumococcal, tetanus toxoid, against HBV and HAV, HPV, etc.) [18, 34].

Still, the dilemma for patients with rheumatoid arthritis, lupus, scleroderma, and other autoimmune conditions exists whether the vaccine's potency will be accomplished or the effectiveness will be diminished. In any case, some vaccines are recommended even when the immune responses and protection are uncertain. And in some cases, the use of antiviral drugs early during the viral infection in patients with AIIDs is a must, although they have been vaccinated (i.e., in case of influenza). For other cases, the increased dosage of influenza vaccine would help counteract the reduced response; or to pause the treatment temporarily or just before the vaccine is administered and resuming after two weeks for patients on methotrexate who are at high risk for influenza [35].

Although the immunogenicity and the effectiveness of the vaccines in this population of patients may be lower than the healthy controls, they are generally comparable. Besides, the data on the vaccine's effectiveness in the rheumatic population of adults and children are insufficient because only a few studies follow up the length of protection and different modalities of the immune responses after vaccination [25].

The greatest concern of people with rheumatic diseases is whether the risk of flare or exacerbation of the disease is higher after vaccination. An analysis of the existing literature up to 2010, including more than 5000, showed that people who already had autoimmune diseases were not endangered by exacerbation or worsening of the disease [23]. The data showed no vaccines raised safety issues or worsening of the disease in children with rheumatoid arthritis (incl. MMR vaccine); multiple sclerosis, reactive arthritis, vasculitides, type 1 diabetes, lupus erythematosus (except HBV and tickborne encephalitis vaccines that were linked to neurological manifestations). Nevertheless, taking into account that due to immunosuppressive therapy, these patients are more prone to infectious disease and severe illness with the development of complications, the benefits of the vaccination outweigh the possibility of disease exacerbation [25].

Patients should ideally be vaccinated during remission or low-disease-activity state of AIID and before planned immunosuppression [18]. However, this might not be possible in a significant proportion of rheumatic patients. In the case of potent immunosuppression, based on the accumulated experience with influenza vaccines, repeated revaccinations may theoretically boost immunogenicity securing higher levels of seroprotection. Furthermore, while on antiCD20 therapy, dose interruption to allow immature B cells to recover may be an option to improve vaccine efficacy [17]. Given the lack of empirical data with COVID-19 vaccines in rheumatic patients, we hypothesize possible modifiers of vaccine response based on extrapolated data from other wellknown non-live vaccines [36-39], summarized in Table 1.

\section{Types of vaccines for COVID-19 and concerns for patients with rheumatic diseases}

The World Health Organization (WHO) has recognized the need for a safe and efficient vaccine to manage the spread of the SARS-CoV-2 infection worldwide during the COVID-19 pandemic [40]. However, patients with rheumatic disease fall in the groups of patients with chronic disease who will be included in the trials of the new COVID-19 vaccines at the next stage. Many questions regarding the immunogenicity, effectiveness, and safety were raised with the development and the progress of the candidates [18]. Currently, 60 vaccines are included in the overall of 152 trials in 41 countries worldwide. The need to address the immediate demand for vaccines allows applying a new model framework for vaccine development. This has led to shortening the developmental timelines of usually $10-15$ years to $1-2$ years [41].

There are a variety of approaches towards building a protective immunity against SARS-CoV-2. Still, the main are four: whole virus (inactivated or weakened), viral vector (replicating and non-replicating), nucleic acid (RNA, DNA), and protein-based (protein subunit, virus-like particle) [42]. All vaccines try to introduce viral antigens to the immune system to obtain effective immune responses that will eliminate or block the virus from entering the cells and cause illness. At least, exposed patients to develop an asymptomatic or mild form of COVID-19.

The vaccines' ultimate goal is to provide long-lasting protection against the virus for rheumatic patients without putting them at risk of flares or complications. Nevertheless, the information for some of the vaccines is still scarce. However, with accumulating data, the decision-making for rheumatologists which patients to vaccinate, when and with what vaccine, will become easier.

Additionally, different vaccines against COVID-19 have distinct logistic issues (i.e., variable stability, transportation in liquid nitrogen, need for more booster doses, etc.) that can hinder their distribution around the globe, especially affecting vaccine availability in low-income countries. This may result in achieving different levels of population immunity against COVID-19 or lack of vaccination at all in some regions.

\section{Whole virus-based anti-COVID-19 vaccines}

The virus-based vaccine candidates use weakened or inactivated virus processed by either conventional technology by passing the virus through animal or human cells leading 
Table 1 Hypothesized modifiers of vaccine response and considerations in patients with autoimmune inflammatory diseases

\begin{tabular}{|c|c|c|}
\hline Potential modifiers & Specific population group & Considerations \\
\hline \multicolumn{3}{|l|}{ General viewpoints } \\
\hline Age & Elderly patients & $\begin{array}{l}\text { Elderly patients may have diminished immune } \\
\text { reactivity and reduced seroconversion rate } \\
\text { due to, in part, the long-standing autoimmune } \\
\text { inflammatory disease with its confounding } \\
\text { effect }\end{array}$ \\
\hline Gender & Male patients & $\begin{array}{l}\text { Male gender may be associated with lower } \\
\text { acceptance rate and lower rates of postvaccina- } \\
\text { tion seroconversion [36] }\end{array}$ \\
\hline Immune history & Exposed to virus or vaccinated patients & $\begin{array}{l}\text { "Immune imprinting" due to prior coronavirus } \\
\text { exposure may affect both positively or nega- } \\
\text { tively vaccine effectiveness [37] }\end{array}$ \\
\hline Comorbidity & Obese and multimorbid patients & $\begin{array}{l}\text { Obesity and end-organ damage may affect host } \\
\text { response to vaccines due to altered T-cell func- } \\
\text { tion and reduced levels of interferon-gamma } \\
\text { and granzyme B [38] }\end{array}$ \\
\hline \multicolumn{3}{|l|}{ Autoimmune disease-specific viewpoints } \\
\hline Disease activity and duration & $\begin{array}{l}\text { Patients with high disease activity and/or long- } \\
\text { standing disease }\end{array}$ & $\begin{array}{l}\text { The long-standing and active autoimmune inflam- } \\
\text { matory disease may affect immunogenicity by } \\
\text { reducing seroconversion rates [39] }\end{array}$ \\
\hline B cell depleting therapy & $\begin{array}{l}\text { Rheumatic patients undergoing CD20 depleting } \\
\text { therapy }\end{array}$ & $\begin{array}{l}\text { B cell responses and thus, a serological response } \\
\text { to vaccines are hindered by CD20 depletion } \\
\text { therapy [17] }\end{array}$ \\
\hline $\begin{array}{l}\text { High-dose glucocorticoids and other } \\
\text { immunosuppressive agents }\end{array}$ & Immunosuppressed patients & $\begin{array}{l}\text { Variable impact on vaccine effectiveness with the } \\
\text { majority of patients having satisfactory serocon- } \\
\text { version rates [18] }\end{array}$ \\
\hline
\end{tabular}

to mutations, or by chemical substances (most commonly formaldehyde and heat) that make it less virulent. The use of whole attenuated virus resembles the natural infections highly; therefore, the immunity includes all the aspects of the immune response (innate and adaptive). However, although very efficient, these vaccines require a longer time to develop, which delays the process. Currently, two inactivated vaccines against SARS-CoV-2 were approved by at least one country: Covaxin (Bharat Biotech) and BBIBPCorV (Sinopharm). The other whole virus-based vaccines with an inactivated virus, such as Inactivated (Sinopharm), CoronaVac (Sinovac), Inactivated (Chinese Academy of Medical Sciences), are still in phase III of clinical trials. The sole live-attenuated COVI-VAC (Codagenic Inc) is in phase I.

Nevertheless, this kind of vaccine raised the most safety concerns. This is especially valid for patients with autoimmune rheumatic disease since they may be immunocompromised mainly due to the treatment. According to current EULAR recommendations, live-attenuated vaccines may be considered with caution in patients with rheumatic diseases; however, their immunocompetent family members should be encouraged to receive inactivated and live-attenuated vaccines according to the national guidelines (e.g., MMR, rotavirus, varicella, and zoster vaccine; except for the oral polio vaccine) [18]. Suppose we extrapolate this postulate on COVID-19 vaccines. In that case, patients with AIIDs may obtain the benefits of other types of vaccines. Still, their relatives may be vaccinated with inactivated/live-attenuated vaccines against SARS-CoV-2 to protect AIIDs patients, especially in the expected vaccine shortage.

\section{Protein subunit-based anti-COVID-19 vaccines}

Protein-based vaccines rely on fragments of viral proteins or proteins that mimic the SARS-CoV-2 outer coat. Most candidate vaccines that use protein subunits focus on the spike $S$ protein, particularly on its receptor-binding domain. Usually, this kind of vaccine requires adjuvants to overcome some of the vaccines' limitations and enhance the immunogenicity of the vaccine. Besides, eventually, they need multiple doses to establish an effective and long-lasting immune response.

A virus-like particle is an approach that utilizes empty virus shells that mimic the coronavirus structure. It is known that this kind of particles can induce a robust immune response. The main limitation is that they are challenging to manufacture.

The candidate vaccines that employ protein subunits in phase III are RBD-Dimer (Anhui Zhifei Longcom), NVXCoV2373 (Novavax), and virus-like particles_-Plant-based 
VLP (Medicago). The other candidates are still in phase I or II of clinical trials. With regards to patients with AIIDs, experience with such vaccines was accumulated mostly for seasonal trivalent subunit influenza vaccination showing good immunogenicity and effectiveness in patients treated with variable disease-modifying rheumatic drugs [43, 44], except for rituximab [45]. Although not investigated in patients with AIIDs, the non-live recombinant subunit adjuvant vaccine against zoster may replace the live-attenuated vaccines [46].

For these reasons, we can assume that COVID-19 vaccines based on protein subunits are potentially among the vaccines with the best safety/effectiveness profile for patients with rheumatic diseases.

\section{Viral vector-based anti-COVID-19 vaccines}

Vaccine platforms using vectors to deliver the virus in the organisms are a well-known approach. For vectors, genetically engineered measles or adenoviruses are used. Thus, they cannot cause disease. They are two types: replicating and non-replicating. Replicating viral vectors (such as weakened measles) against COVID-19 can replicate after entering the host cells; however, there is no risk for infection occurrence-neither of the measles, not of the SARS-CoV-2. The vaccine-induced immune response tends to be strong, with an excellent safety profile of the vaccine. Like the other viral vectors for SARS-CoV-2 antigens, adenovirus cannot replicate. Still, it can deliver coronavirus spike or other genes in the host cells. Although not used in a licensed vaccine, adenoviruses have a long history in gene therapy.

However, the main drawback of vaccines based on viral vectors is the low effectiveness due to prior existing immune response to the vector and the need for frequent booster doses for establishing long-lasting immune response. Available data on vector-based vaccines in immunocompromised patients suggest that disseminated infection is highly unlikely. Importantly, viral vector replication is incompetent and viral-based vaccines are well tolerated. Among the solicited and unsolicited adverse events, severe ones were not observed in a host with a compromised immune system [47].

At the time of writing the paper, the following nonreplicating viral vector vaccines Sputnik V (Gamaleya), AZD1222 (Oxford/AstraZeneca), and Covishield (Serum Institute of India) were approved by at least one country. In phase III are Ad5-nCoV (CanSino) and Ad26.COV2.S (Janssen) so far. Vaccines based on replicating viral vectors, such as IIBR-100 (Israel Institute for Biological Research) and DeINS1-2019-nCoV-RBD-OPT (Wantaj), are still in phase II.

To date, there is no experience of viral-vector based vaccines against infectious agents in patients with AIIDs, although there are approaches for viral-vector gene therapy in patients with immune-mediated diseases [48]. Nevertheless, three cases of transverse myelitis were reported after ChAdOx1 nCoV-19 (AZD1222) booster vaccination. Initially, they were described as potentially related to the vaccination unexpected serious adverse reactions. Later, they were considered by independent neurological experts as "idiopathic" spinal cord demyelination or "pre-existing", but previously unrecognized, multiple sclerosis. The relationship between the vaccine and acute transverse myelitis remained possible in only one of the cases [49]. All three trial participants have improved or recovered. Independent clinical review of the safety data showed an acceptable safety profile and effectiveness against symptomatic COVID-19. The monitoring of safety focused on neurological adverse events continues in the trials.

\section{Nucleic acid}

The majority of vaccine platforms that implement nucleic acids use instructions to synthesize the $\mathrm{S}$ protein of SARSCoV-2 (in the form of RNA or DNA). By a process called electroporation, the uptake of DNA instructs into the host cells is enhanced. In contrast, RNA-based vaccines include lipid nanoparticles to allow RNA to enter the host cells. The advantage of these approaches is the fast and facilitated development with a low-to-moderate manufacturing scale due to the production of the genetic instructs but not the viral proteins.

However, the major drawback is that there are no licensed vaccines that use this technology so far, although mRNA vaccines (for influenza, rabies, and Zika virus) have been previously verified in animals. Besides mRNA vaccines against anti-infectious agents, mRNA technology was successfully implemented in cancer vaccines (incl. dendritic cells, directly injectable mRNA, etc.), which have been tested in many cancer clinical trials, with promising results in eliciting strong $\mathrm{T}$ cell responses and increased diseasefree survival of patients [50-52].

However, as the novel and potent for rapid spread pathogens such as SARS, Ebola, and Zika arise, the need for global preparedness for pandemics has been highlighted. This includes the readiness for fast development and distribution of vaccines [53]. Amongst the disadvantages is the logistic issues due to the need for special temperature conditions for the not so stable RNA.

On the COVID-19 vaccines timeline, RNA-based BNT162b2 (BionTech/Pfizer) was approved in 48 countries, and mRNA-1273 (Moderna) - in two countries so far. Another RNA-based vaccine in phase III is $\mathrm{CVnCoV}$ (Curevac). DNA-based vaccines INO-4800 (Inovio) and AG0301COVID-19 (AnGes) are in phase III.

A recent paper by Polack et al. demonstrated 95\% effectiveness of the BNT162b2 vaccine in preventing 
SARS-CoV-2 infection with an excellent safety profile and mostly mild-to-moderate adverse events, comparable with the placebo group and other viral vaccines [54].

Although patients with rheumatic diseases will be considered potential participants in phase III and beyond, this kind of vaccine is generally considered safe for this population. However, only the lack of data here increases the hesitancy amongst the rheumatologists and other specialists to recommend these vaccines for their patients.

Although there is no real-world experience with patients living with AIIDs, some potential advantages of RNA-based vaccines for this specific group should be taken into account. Recent studies demonstrated that mRNA vaccines surpass others, such as live-attenuated, protein subunits, inactivated, and DNA vaccines [50]. In the first place, mRNA is noninfectious, non-integrating vector/instruct. The possibility of contamination or insertional mutagenesis is not even theoretically present. The half-life of mRNA is limited, thus, controlled by the normal cellular processes and different modifications. To further improve the protection profile, the intrinsic immunogenicity of mRNA can be decomposed. Regarding effectiveness, a vast number of improvements could be done to make mRNA highly translatable and enable rapid cytoplasm uptake and expression [55-58]. Some of the possible modifications, such as the incorporation of modified nucleosides, can increase mRNA translation but reduce innate immune activation while preserving production of type I interferons and proinflammatory cytokines [59]

For the patients with rheumatic diseases, the theoretical risk for relapse of the autoimmune disease is related to mRNA's properties. By RNA sensor recognition in dendritic cells, mRNA from the vaccines can exert adjuvant-life effects on the innate immune system (Fig. 1). It was shown as early as the 60's in the last century that single-stranded mRNAs are pathogen-associated molecular patterns that activate toll-like receptors TLR7 and TLR8, resulting in type I interferon production [60]. A study from 2017 showed that mRNA vaccines could increase cytokines and chemokines' production after intradermal application.

However, mRNA instruction may be modified not to activate TLRs, thus decreasing IFN type I secretion [61].

By stimulating dendritic cells' maturation and eliciting robust $\mathrm{T}$ and $\mathrm{B}$ cell responses, RNA-based vaccines may activate bystander autoreactive lymphocytes. Theoretically, this process can reactivate autoimmune diseases. However, mRNA exhibits an inhibiting function on antigen expression that may suppress the immune response $[55,59]$. Therefore, we cannot predict the overall outcomes from the mRNA action on innate and adaptive immunity through vaccines
[62]. All the paradoxical effects of mRNA on innate immune sensing have to be elucidated, in vivo and clinically $[55,59]$.

However, we have to emphasize that the innate responses after vaccination with RNA-based vaccines are not the primary responses. In line with this, effects of RNA-based vaccines on the innate immunity are complementary. On the one hand, these effects are desirable, considering the viral mechanisms of inhibiting interferons and other arms of the immune system. On the other hand, the activation of the innate immune system may contribute to the non-specific activation of autoreactive lymphocytes leading to higher risk of relapse of the current disease or hypothetically developing an AIID.

To the best of our knowledge, real-world data on COVID19 vaccines in patients with AIIDs tend to zero. The exclusion criteria for the two currently approved mRNA vaccines at different stages of the study included autoimmune diseases. Interestingly, the US Food and Drug Administration (FDA) considered that there was a possibility that the vaccine had contributed to the onset of rheumatoid arthritis [63].

\section{Conclusion}

Establishing proper recommendations for the newly developed vaccines against SARS-CoV-2 would be challenging. Although vaccine administration has been associated with autoimmune manifestations in certain genetically predisposed individuals, it was demonstrated that vaccinations do not pose a more prominent danger than natural infections themselves.

Both patients and clinicians are concerned about the potential risk for relapse or worsening of autoimmune diseases mainly because of insufficient data. At this stage of the trials, patients with rheumatic diseases are included only by exception. Clinical data extracted from these single cases cannot be applied to all patients with AIIDs. Experience with well-known vaccines can provide guidance and partial confidence for the use of the "new vaccines". Still, AIID patients need to be included in the ongoing clinical trials to ensure vaccine safety and efficacy in the context of an autoimmune disease.

The associated vaccination risk should not lead to vaccine refusal or major delay in rheumatic patients who will soon have at their disposal different vaccine platforms with variable safety and cost-effectiveness profiles. Furthermore, trials would clarify the underlying immunological mechanisms of the newly implemented vaccines/adjuvants in the AIIDs population. 


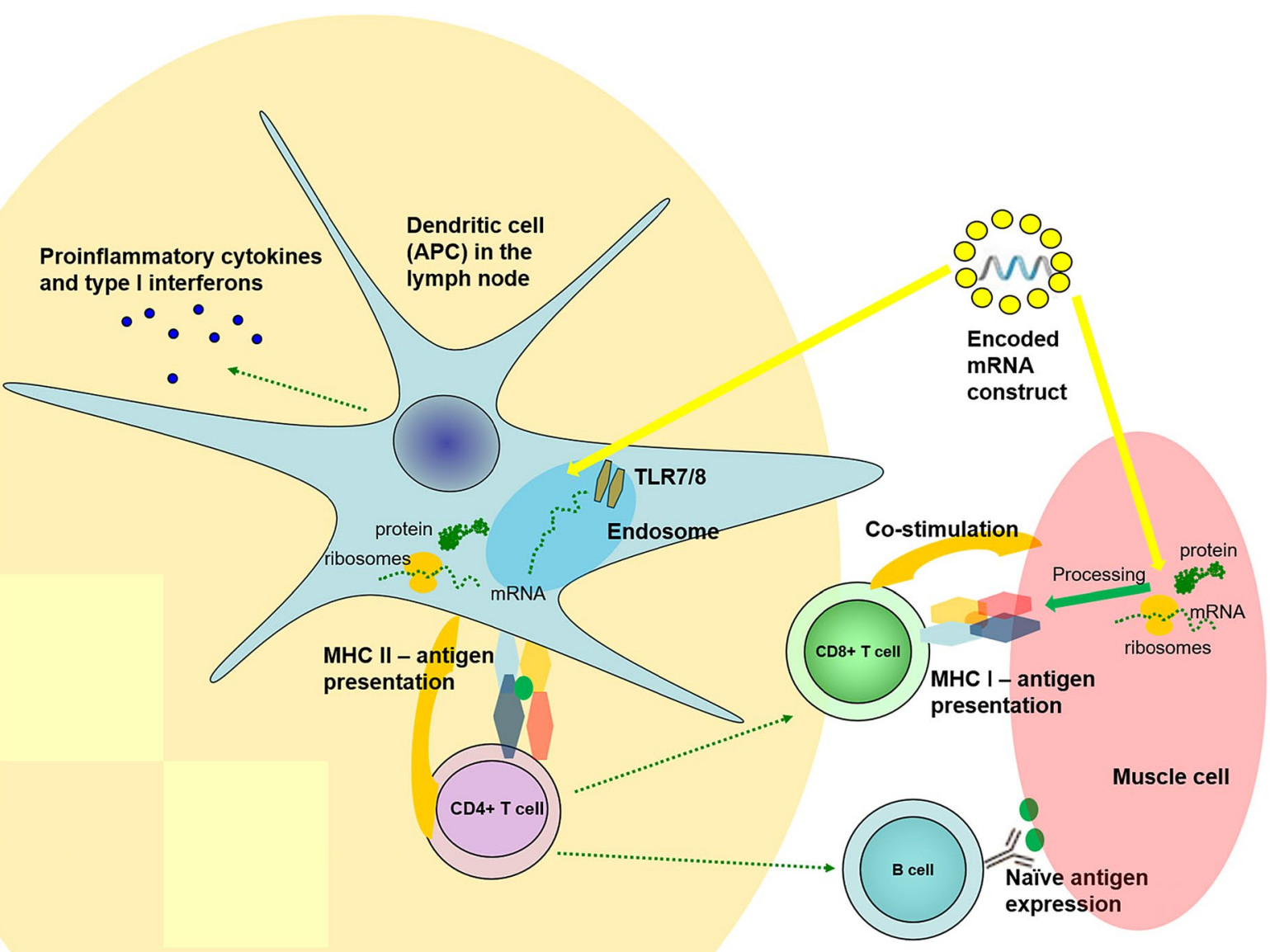

Fig. 1 The immune processes involved in the mechanism of mRNA vaccines-activation of T helper cells (CD4+) via MHC I molecules and processed viral antigen in antigen-presenting cells in the lymph node; stimulation of $\mathrm{T}$ cytotoxic cells (CD8+) via MHC class II molecules and processed viral antigen and $\mathrm{B}$ cell by native viral antigens.
In antigen-presenting cells, mRNA sense TLR7 and 8, leading to activation of down cascade and production and secretion of proinflammatory cytokines and type I interferons. Some of the mechanisms are simplified in the figure by omitting (i.e., the inflammasome, the proteasome, secondary messengers, etc.)
Acknowledgements We acknowledge the work of Ivalina Alexieva and Aleksandar Kopchev in providing writing assistance, language editing, and proofreading of the final manuscript version. We thank our families for providing us the time and support to write this manuscript in a timely manner.

Author contributions Both TV and TG were involved in the conception and design of the manuscript, database search process, analysis, and logical interpretation. The manuscript was critically revised and its final version was approved by both authors for important intellectual content. TV and TG agreed to be accountable for all aspects of the work.
Funding No funding was received for this study.

\section{Compliance with ethical standards}

Conflict of interest The authors declare that there is no conflict of interest regarding the publication of this article.

Ethical approval This article does not contain any studies with human participants or animals performed by any of the authors. 


\section{References}

1. Lambert H, Gupte J, Fletcher H, Hammond L, Lowe N, Pelling M et al (2020) COVID-19 as a global challenge: towards an inclusive and sustainable future. Lancet Planet Health 4(8):e312-314

2. Haug N, Geyrhofer L, Londei A, Dervic E, Desvars-Larrive A, Loreto V, Pinior B, Thurner S, Klimek P (2020) Ranking the effectiveness of worldwide COVID-19 government interventions. Nat Hum Behav 4(12):1303-1312

3. Gasparyan AY, Misra DP, Yessirkepov M, Zimba O (2020) Perspectives of immune therapy in Coronavirus disease 2019. J Korean Med Sci 35(18):e176

4. Georgiev T, Angelov AK (2020) Complexities of diagnosis and management of COVID-19 in autoimmune diseases: potential benefits and detriments of immunosuppression. World J Clin Cases 8(17):3669-3678

5. Fontanet A, Cauchemez S (2020) COVID-19 herd immunity: where are we? Nat Rev Immunol 20(10):583-584

6. Saxena S, Skirrow H, Bedford H (2020) Routine vaccination during covid-19 pandemic response. BMJ 369:m2392

7. Burgess RA, Osborne RH, Yongabi KA, Greenhalgh T, Gurdasani D, Kang G et al (2020) The COVID-19 vaccines rush: participatory community engagement matters more than ever. Lancet S0140673620326428

8. Global Alliance for Vaccines and Immunizations (GAVI) (2020) The latest in the COVID-19 vaccine race. Available from: https ://www.gavi.org/vaccineswork/covid-19-vaccine-race. Accessed 14 Dec 2020

9. Gasparyan AY, Ayvazyan L, Blackmore H, Kitas GD (2011) Writing a narrative biomedical review: considerations for authors, peer reviewers, and editors. Rheumatol Int 31(11):1409-1417

10. Theofilopoulos AN, Kono DH, Baccala R (2017) The multiple pathways to autoimmunity. Nat Immunol 18(7):716-724

11. Galeotti C, Bayry J (2020) Autoimmune and inflammatory diseases following COVID-19. Nat Rev Rheumatol 16(8):413-414

12. Misra DP, Agarwal V, Gasparyan AY, Zimba O (2020) Rheumatologists' perspective on Coronavirus disease 19 (COVID-19) and potential therapeutic targets. Clin Rheumatol 39(7):2055-2062

13. European League Against Rheumatism (EULAR) (2020) EULAR-COVID-19 Database. Available from https://www. eular.org/eular_covid19_database.cfm. Accessed 19 Dec 2020

14. Kłodziński Ł, Wisłowska M (2018) Comorbidities in rheumatic arthritis. Reumatologia 56(4):228-233

15. Kipps S, Paul A, Vasireddy S (2020) Incidence of COVID-19 in patients with rheumatic disease: is prior health education more important than shielding advice during the pandemic? Clin Rheumatol 11:1-5

16. Serling-Boyd N, D'Silva KM, Hsu TY, Wallwork R, Fu X, Gravallese EM et al (2020) Coronavirus disease 2019 outcomes among patients with rheumatic diseases 6 months into the pandemic. Ann Rheum Dis

17. Baker D, Roberts CAK, Pryce G, Kang AS, Marta M, Reyes $S$ et al (2020) COVID-19 vaccine-readiness for anti-CD20depleting therapy in autoimmune diseases. Clin Exp Immunol 202(2):149-161

18. Furer V, Rondaan C, Heijstek MW, Agmon-Levin N, van Assen S, Bijl M et al (2020) 2019 update of EULAR recommendations for vaccination in adult patients with autoimmune inflammatory rheumatic diseases. Ann Rheum Dis 79(1):39-52

19. Ahmed S, Gasparyan AY, Zimba O (2021) Comorbidities in rheumatic diseases need special consideration during the COVID-19 pandemic. Rheumatol Int 41:243-256
20. Munz C, Lunemann JD, Getts MT, Miller SD (2009) Antiviral immune responses: triggers of or triggered by autoimmunity? Nat Rev Immunol 9:246-258

21. Agmon-Levin N, Paz Z, Israeli E, Shoenfeld Y (2009) Vaccines and autoimmunity. Nat Rev Rheumatol 5:648-652

22. Schattner A (2005) Consequence or coincidence? The occurrence, pathogenesis and significance of autoimmune manifestations after viral vaccines. Vaccine 23:3876-3886

23. Salemi S, D'Amelio R (2010) Could autoimmunity be induced by vaccination? Int Rev Immunol 29:47-69

24. Vadalà M, Poddighe D, Laurino C, Palmieri B (2017) Vaccination and autoimmune diseases: is prevention of adverse health effects on the horizon? EPMA J 8(3):295-311

25. De Martino M, Chiappini E, Galli L (2013) Vaccines and autoimmunity. Int J Immunopathol Pharmacol 26(2):283-290

26. Rodríguez Y, Rojas M, Pacheco Y, Acosta-Ampudia Y, RamírezSantana C, Monsalve DM et al (2018) Guillain-Barré syndrome, transverse myelitis and infectious diseases. Cell Mol Immunol 15(6):547-562

27. Markovic-Plese S, Hemmer B, Zhao Y, Simon R, Pinilla C, Martin R (2005) High level of cross-reactivity in influenza virus hemagglutinin-specific CD4+ T cell response: implications for the initiation of autoimmune response in multiple sclerosis. J Neuroimmunol 169(1-2):31-38

28. Sioofy-Khojine AB, Lehtonen J, Nurminen N, Laitinen OH, Oikarinen S, Huhtala $\mathrm{H}$ et al (2018) Coxsackievirus B1 infections are associated with the initiation of insulin-driven autoimmunity that progresses to type 1 diabetes. Diabetologia 61(5):1193-1202

29. Takahashi Y, Murai C, Shibata S, Munakata Y, Ishii T, Ishii K et al (1998) Human parvovirus B19 as a causative agent for rheumatoid arthritis. Proc Natl Acad Sci USA 95(14):8227-8232

30. Institute of Medicine (2011) Adverse effects of vaccines: evidence and causality. The National Academies Press, Washington DC

31. Kivity S, Agmon-Levin N, Blank M, Shoenfeld Y (2009) Infections and autoimmunity-friends or foes? Trends Immunol 30:409-414

32. Rioux JD, Abbas AK (2005) The genetics of complex autoimmune diseases. Nature 435:584-589

33. Chen RT, Pless R, De Stefano F (2001) Epidemiology of autoimmune reactions induced by vaccination. J Autoimmun 16(3):309-318

34. Szwejkowska M, Kuchar E (2020) Vaccinations in rheumatic diseases. Reumatologia 58(2):61-62

35. Colmegna I, Useche ML, Rodriguez K, McCormack D, Alfonso G, Patel A et al (2019) Immunogenicity and safety of high-dose versus standard-dose inactivated influenza vaccine in rheumatoid arthritis patients: a randomised, double-blind, active-compatator trial. Lancet Rheum 2(1):E14-E23

36. Flanagan KL, Fink AL, Plebanski M, Klein SL (2017) Sex and gender differences in the outcomes of vaccination over the life course. Annu Rev Cell Dev Biol 33:577-599

37. Dhakal S, Klein SL (2019) Host factors impact vaccine efficacy: implications for seasonal and universal influenza vaccine programs. J Virol 93(21):e00797-e819

38. Sheridan PA, Paich HA, Handy J, Karlsson EA, Hudgens MG, Sammon AB et al (2012) Obesity is associated with impaired immune response to influenza vaccination in humans. Int J Obes 36:1072-1077

39. Campos LM, Silva CA, Aikawa NE, Jesus AA, Moraes JC, Miraglia J et al (2013) High disease activity: an independent factor for reduced immunogenicity of the pandemic influenza a vaccine in patients with juvenile systemic lupus erythematosus. Arthritis Care Res (Hoboken) 65(7):1121-1127

40. World Health Organization (2020) Draft landscape of COVID19 candidate vaccines. Available from https://www.who.int/publi 
cations/m/item/draft-landscape-of-covid-19-candidate-vaccines. Accessed 18 Dec 2020

41. Lurie N, Saville M, Hatchett R, Halton J (2020) Developing COVID-19 vaccines at pandemic speed. N Engl J Med 382(21):1969-1973

42. Le Thanh T, Andreadakis Z, Kumar A, Gómez Román R, Tollefsen S, Saville M, Mayhew S (2020) The COVID-19 vaccine development landscape. Nat Rev Drug Discov 19(5):305-306

43. Chang CC, Chang YS, Chen WS, Chen YH, Chen JH (2016) Effects of annual influenza vaccination on morbidity and mortality in patients with systemic lupus erythematosus: a nationwide cohort study. Sci Rep 6:37817

44. Kostianovsky A, Charles P, Alves JF, Goulet M, Pagnoux C, Le Guern V et al (2012) French Vasculitis Study Group. Immunogenicity and safety of seasonal and 2009 pandemic A/H1N1 influenza vaccines for patients with autoimmune diseases: a prospective, monocentre trial on 199 patients. Clin Exp Rheumatol 30:S83-S89

45. Oren S, Mandelboim M, Braun-Moscovici Y, Paran D, Ablin J, Litinsky I et al (2008) Vaccination against influenza in patients with rheumatoid arthritis: the effect of rituximab on the humoral response. Ann Rheum Dis 67(7):937-941

46. Lal H, Cunningham AL, Godeaux O, Chlibek R, Diez-Domingo J, Hwang SJ et al (2015) Efficacy of an adjuvanted herpes zoster subunit vaccine in older adults. N Engl J Med 372(22):2087-2096

47. Custers J, Kim D, Leyssen M, Gurwith M, Tomaka F, Robertson J et al (2020) Vaccines based on replication incompetent Ad26 viral vectors: standardized template with key considerations for a risk/ benefit assessment. Vaccine

48. Voysey M, Clemens SAC, Madhi SA, Weckx LY, Folegatti PM, Aley PK et al (2020) Safety and efficacy of the ChAdOx1 nCoV19 vaccine (AZD1222) against SARS-CoV-2: an interim analysis of four randomised controlled trials in Brazil, South Africa, and the UK. Lancet 397(10269):99-111

49. De Haan P, Van Diemen FR, Toscano MG (2020) Viral gene delivery vectors: the next generation medicines for immunerelated diseases. Hum Vaccin Immunother 15:1-8

50. Pardi N, Hogan MJ, Porter FW, Weissman D (2018) mRNA vaccines-a new era in vaccinology. Nat Rev Drug Discov 17(4):261-279

51. Weissman D (2015) mRNA transcript therapy. Expert Rev Vaccines 14:265-281

52. Sahin U, Kariko K, Tureci O (2014) mRNA-based therapeutics-developing a new class of drugs. Nat Rev Drug Discov 13:759-780
53. Rauch S, Jasny E, Schmidt KE, Petsch B (2018) New vaccine technologies to combat outbreak situations. Front Immunol 9:1963

54. Polack FP, Thomas SJ, Kitchin N, Absalon J, Gurtman A, Lockhart S et al (2020) Safety and efficacy of the BNT162b2 mRNA COVID-19 vaccine. N Engl J Med 383:2603-2615

55. Karikó K, Muramatsu H, Welsh FA, Ludwig J, Kato H, Akira S, Weissman D (2008) Incorporation of pseudouridine into mRNA yields superior nonimmunogenic vector with increased translational capacity and biological stability. Mol Ther 16(11):1833-1840

56. Kauffman KJ, Webber MJ, Anderson DG (2016) Materials for non-viral intracellular delivery of messenger RNA therapeutics. J Control Release 240:227-234

57. Guan S, Rosenecker J (2017) Nanotechnologies in delivery of mRNA therapeutics using nonviral vector-based delivery systems. Gene Ther 24:133-143

58. Thess A, Grund S, Mui BL, Hope MJ, Baumhof P, Fotin-Mleczek M, Schlake T (2015) Sequence-engineered mRNA without chemical nucleoside modifications enables an effective protein therapy in large animals. Mol Ther 23(9):1456-1464

59. Kariko K, Muramatsu H, Ludwig J, Weissman D (2011) Generating the optimal mRNA for therapy: HPLC purification eliminates immune activation and improves translation of nucleoside-modified, protein-encoding mRNA. Nucleic Acids Res 39:e142

60. Isaacs A, Cox RA, Rotem Z (1963) Foreign nucleic acids as the stimulus to make interferon. Lancet 2:113-116

61. Edwards DK, Jasny E, Yoon H, Horscroft N, Schanen B, Geter $\mathrm{T}$ et al (2017) Adjuvant effects of a sequence-engineered mRNA vaccine: translational profiling demonstrates similar human and murine innate response. J Transl Med 15(1):1

62. Jeyanathan M, Afkhami S, Smaill F, Miller MS, Lichty BD, Xing Z (2020) Immunological considerations for COVID-19 vaccine strategies. Nat Rev Immunol 20(10):615-632

63. US Food and Drug Administration (FDA) (2020) Vaccines and Related Biological Products Advisory Committee Meeting. FDA Briefing Document Moderna COVID-19 Vaccine. Available from https://www.fda.gov/media/144434/download. Accessed 20 Dec 2020

Publisher's Note Springer Nature remains neutral with regard to jurisdictional claims in published maps and institutional affiliations. 